\title{
Development of a communication interface to determinate the thermal diffusivity as a function of temperature by infrared thermography
}

by P.R. Correa*, T.M. Pereira*, M.N. Veloso* and D.M. Zezell*

\author{
${ }^{*}$ Center for Lasers and Applications, IPEN-CNEN/SP, Av. Prof. Lineu Prestes 2242, São Paulo, \\ SP, Brazil 05508-000, correa.paulo@gmail.com
}

\begin{abstract}
The aim of this work is to development a software (CZ ThermaDiff) to determinate the thermal diffusivity from biomaterials, like enamel, using infrared thermography. This software was develop in graphical programming in LabView (National Instruments), integrating the infrared camera (Thermacam SC3000, FLIR) to make the system easier and comfortable to any user, besides their knowledge about the equipment. In this first experiment it was possible to plot the thermal diffusivity of enamel as a function of temperature, but for further experiments the thermal diffusivity of different biomaterials will be obtained.
\end{abstract}

\section{Introduction}

Lasers have been used in many dentistry proceedings that can cause an increase in temperature in the tissue itself or in its adjacent. That increase could cause reversible or irreversible damage[1], such as tooth necrosis, since the temperature of the tooth pulp can not increase $5.5^{\circ} \mathrm{C}[2]$. The tooth coronal potion is divided in three layers: enamel, dentin and pulp, than the enamel is mineralized tissue compound mainly by $96 \%$ of hydroxyapatite and $4 \%$ of organic matrix[3]. The dentin is quite similar than enamel, main difference is the organic matrix is much higher than in enamel and the dentin is thicker than enamel, also the dentin have the extension of specialized (odontoblasts) cells from de pulp, where is located the nerve endings and blood vessels[4].

The thermal diffusivity can be measured by several methods, for example, photoacustic method[5], Angstron method[6] and laser flash method[7], but these methods were used just at room temperature. A few authors have used the infrared thermography method to calculate the thermal diffusivity[8,4]. Thus in this work we used the infrared thermography method to calculate the thermal diffusivity of enamel and dentin in high temperatures through the heat equation[9]. The enamel when is irradiated by laser, there is a drastic increase of temperature and in high temperatures it can suffer chemical and physical changes[10].

LabView is easy to understand and can thus be used to achieve productive results more quickly. With LabView, programming is done via block diagrams that consist of icons and wires that can be directly compiled into executable code and at the same time the control panel is built, that mimic a real control panel making the communication with the used easier [11].

\section{Material and methods}

The CZ ThermaDiff's interface was development at LabView (National Instruments, Austin, TX) to become the use of the thermographic camera easier and was development an arrangement to put in line the camera (Thermacam SC 3000, FLIR System, USA), the laser (Er:Cr:YSGG, $\lambda=2,79 \mu \mathrm{m}$ ) and the sample. The sample was positioned in the camera focus at $2 \mathrm{~cm}$ and the laser in the irradiation position (figure 1). The laser was used to heat the sample to high temperatures. 


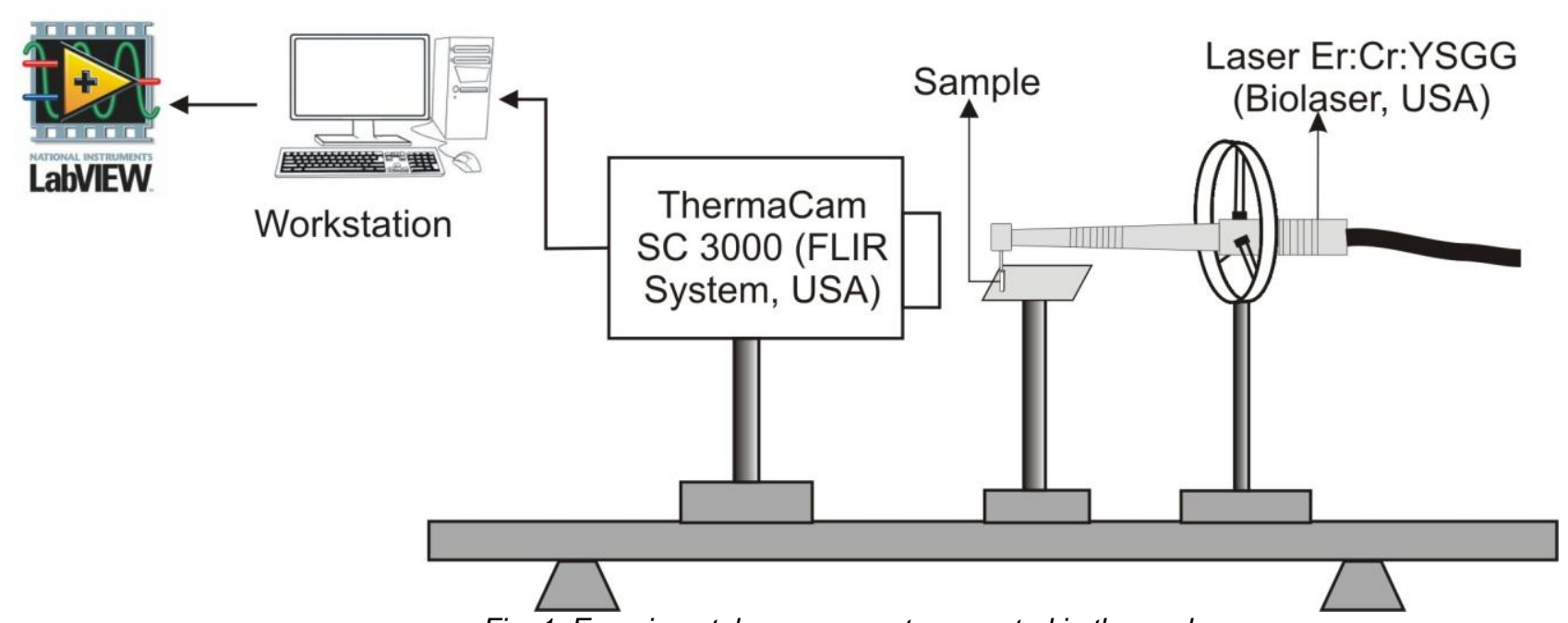

Fig. 1. Experimental arrangement suggested in the work.

\subsection{Sample preparation}

Before the irradiation the samples were prepared, first the tooth were cut to separate the enamel from the dentin. The enamel was cut in blocks of $4 \times 4 \times 1 \mathrm{~mm}$. There was irradiated five samples for each biomaterial. All the samples were cut in the cutter Accutom - 5 (Struers, Denmark) and polished in the polisher EcoMet 250 Grinder-Polisher (Buehler, Germany). In this work were used bovine teeth, these were chosen because the tooth is bigger than a human tooth so it was easier to prepare and easier to find the number of samples that we needed.

\subsection{Irradiation and acquisition}

Thereafter the samples were ready to be irradiated using the laser Er:Cr:YSGG model Waterlase (Biolase Technology, USA), that emits at a wavelength of $2.78 \mu \mathrm{m}$ operates in pulsed mode with temporal width of between 140 $200 \mu \mathrm{s}$ and the repetition rate of $20 \mathrm{~Hz}$. For the irradiation we used the sapphire G6 tip (600 $\mu \mathrm{m}$ of diameter - Biolase Technology, USA). The tip was positioned perpendicular to the sample, the distance from the tip to samples was $1 \mathrm{~mm}$ and only half of the beam reaches the sample. The irradiation time was $10 \mathrm{~s}$ and was used power of $3.75 \mathrm{~W}$ and energy of $98.7 \mathrm{~mJ}$ for all samples.

The acquisition was made by a thermographic camera Thermacam SC 3000(FLIR System, USA), which was developed for research and can work in high acquisition frequency. For this work we used the acquisition frequency of $300 \mathrm{~Hz}$ with a thermal range of $37^{\circ} \mathrm{C}$ to $560^{\circ} \mathrm{C}$ and focal distance of $2 \mathrm{~cm}$.

The work first idea was to integrate the image acquisition to the software that was development by this work, but for some software limitation that was not possible, because the acquisition frequency do not reach the $300 \mathrm{~Hz}$. So first the image sequence was saved in the camera`s software (ThermaCAM Reasearcher, FLIR System/USA) and after load by our software.

For start the acquisition some parameters were given such as the material emissivity, atmospheric temperature, humidity, reflected temperature and sample distance (table 1). When the camera is set to work with an acquisition frequency of $300 \mathrm{~Hz}$ the images have the size of 320x48 pixels and the sequence of images was saved in .SEQ format to be load in the CZ ThemaDiff software.

Tabla 1. Parameters used for the image acquisition by the thermographic camera

\begin{tabular}{|l|l|}
\hline Parameters & Value \\
\hline Material emissivity & 0.91 (for both materials) \\
\hline Humidity & $44 \%$ \\
\hline Atmospheric temperature & $22^{\circ} \mathrm{C}$ \\
\hline External optics temperature & $17^{\circ} \mathrm{C}$ \\
\hline Samples distance & $2 \mathrm{~cm}$ \\
\hline
\end{tabular}




\subsection{Thermal diffusivity calculation}

First to calculate de thermal diffusivity we solve the heat transfer equation (Eq. 1) for the boundary conditions of this work, which were the cylindrical shape of the laser beam and the heat distribution considered was radial. The particular solution is (Eq. 2):

$$
\begin{aligned}
& \frac{\partial T}{\partial t}=\alpha \nabla^{2} T \\
& T(r, t)=\frac{\gamma I_{0} R^{2}}{R^{2}+8 \alpha t} \cdot e^{-\frac{2 r^{2}}{R^{2}+8 \alpha t}}
\end{aligned}
$$

where: $I_{0}$ is the intensity at the center of the laser beam, $\alpha$ is the thermal diffusivity, $t$ is the time, $r$ is the radial variable, $R$ is the radius when the laser beam energy decreases $13.57 \% . \gamma$ is an arbitrary constant.

From the Eq. 2 was obtained Eq. 3:

$$
\varphi(t)=R^{2}+8 \alpha t
$$

Now replacing the Eq. 3 in the Eq. 2 is obtained the Eq. 4:

$$
T(r, t)=\frac{\gamma I_{0} R^{2}}{\varphi(t)} \cdot e^{-\frac{2 r^{2}}{\varphi(t)}}
$$

After this adjustment and with the experimental data was possible to calculate the value of $\varphi(t)$ by comparing the Eq. 4 to the Eq. 5 that is the Gaussian fit, which was made in the experimental data.

$$
f(x)=a e^{-\frac{(x-\mu)^{2}}{2 \sigma^{2}}}+c
$$

Comparing Eq. 4 with Eq. 5 obtains Eq. 6:

$$
-\frac{(r-\mu)^{2}}{2 \sigma^{2}}=-\frac{2 r^{2}}{\varphi(t)}
$$

Where $a$ is the amplitude, $x$ is the radial variable, $\sigma$ standart derivation, $c$ is the offset and $\mu$ is the Gaussian center. One boundary condition is that the $\mu$ is equal to zero, because the point with higher value always is the Gaussian center.

Plotting the values of $\varphi(t)$ as function of the temperature and making a linear fitting (Eq. 7) was possible to compare the Eq. 3 with the linear fitting equation.

$$
f(x)=a x+b
$$

The thermal diffusivity can be obtained by the slope of this line (Eq. 8).

$$
a=8 \alpha
$$

Where $a$ is the slope and $b$ is the intercept of the line. 


\subsection{Software development}

The software CZ ThermaDiff was developed in LabView (National Instruments, Austin, TX) and for the communication between the thermographic camera and the LabView we bought a toolkit (ThermoVision Digital Toolkit 3.1 SR-1) from FLIR. The software works like is shown in figure 2:

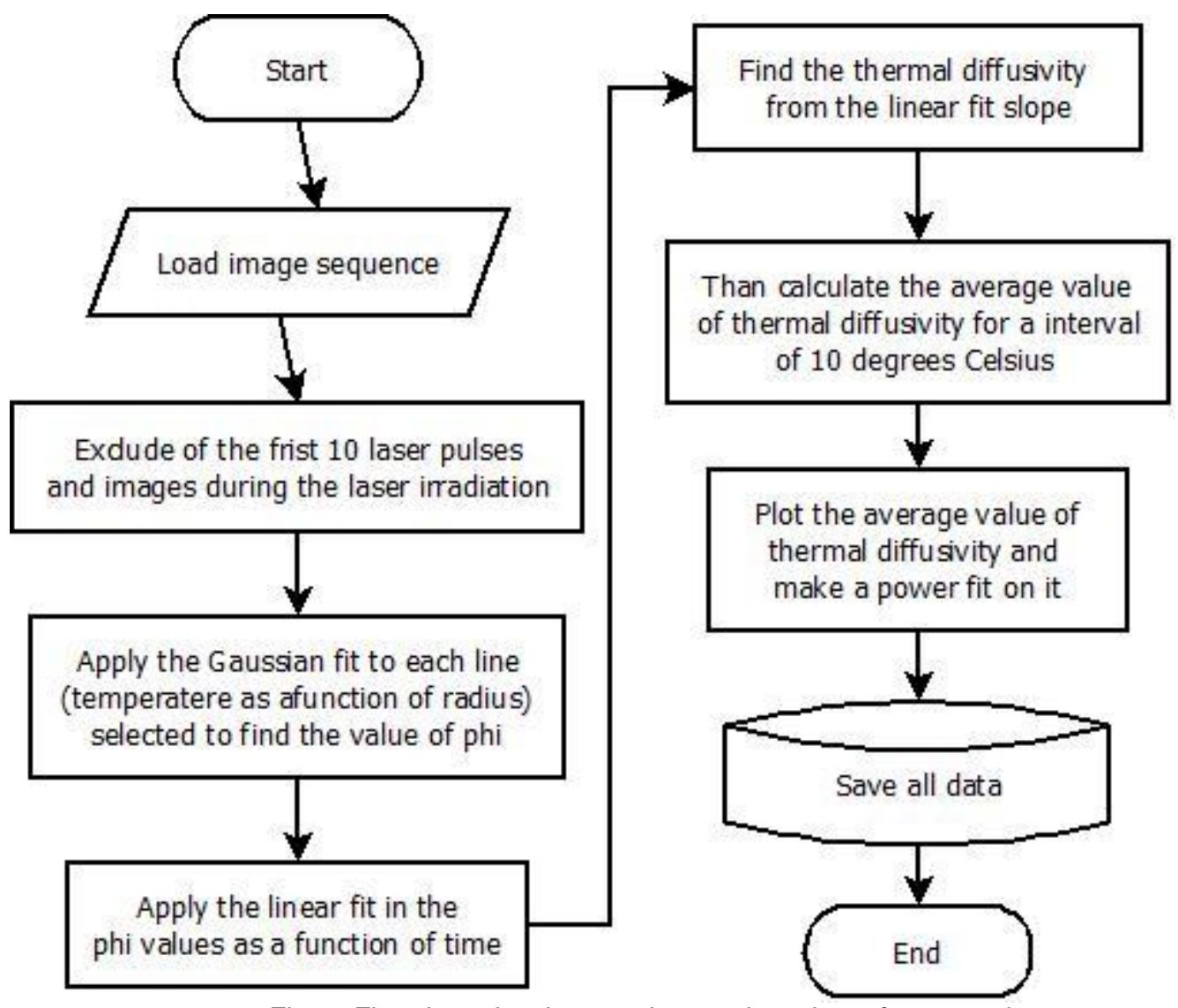

Fig. 2. Flowchart showing step by step how the software works

As we can see in the figure 2 the software start loading the image and save data to manipulation. So the image sequence have some frames before to start the laser irradiation, although the algorithm make a test to find the first frame with the temperature greater than $38{ }^{\circ} \mathrm{C}$, that means the frame where happens the laser irradiation. From this point we start to separate the frames during the laser irradiation from the cooling time. The laser pulse is too short (about $140-$ $200 \mu \mathrm{s})$ for the thermographic camera frequency $(300 \mathrm{~Hz})$ make more than one image and the cooling time is bigger than the laser pulse, it is $46.2 \mathrm{~ms}$ so the number of frame in the cooling time is 14 . Then for more adjustments we just work with the last 10 frames, because in the first 4 frames the number of pixels with the highest temperature is big, so that difficult the Gaussian fitting.

In the second part of the algorithm was made the Gaussian and linear fit on the thermographic data. The software first find transform the images in an array and after for each image the software find the line with highest temperature and create an array with the temperature and the radius distance, each one in one line of the array. The radius distance calculated by the pixel size, which have the size of $0.0018 \mathrm{~cm}$. The software plot this array and make the Gaussian fit (figure 3) and comparing the heat Eq. 4 with the fit Eq. 5 we could find the values of $\varphi(t)$ for each line. So in one frame this happens in 8 consecutives lines and with these $8 \varphi(t)$ values the software build a array with these values with the time values. Therefore the software make a linear fit in this array (figure 4) and compare the fit Eq. 7 with the Eq. 3, so with the fit slope value the software can determinate the thermal diffusivity value. 


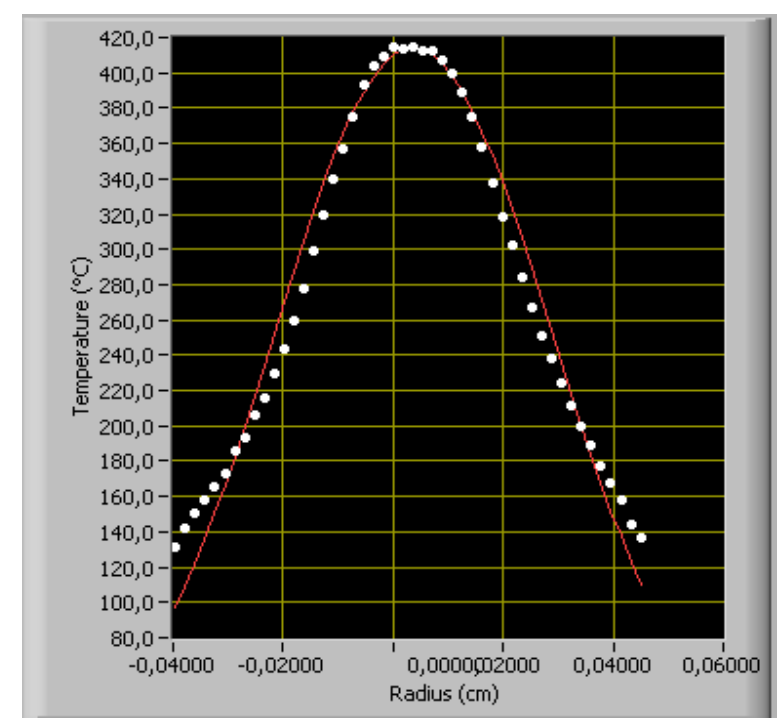

Fig. 3. Temperature array plot indicating by the dots and the Gaussian fit by the red line

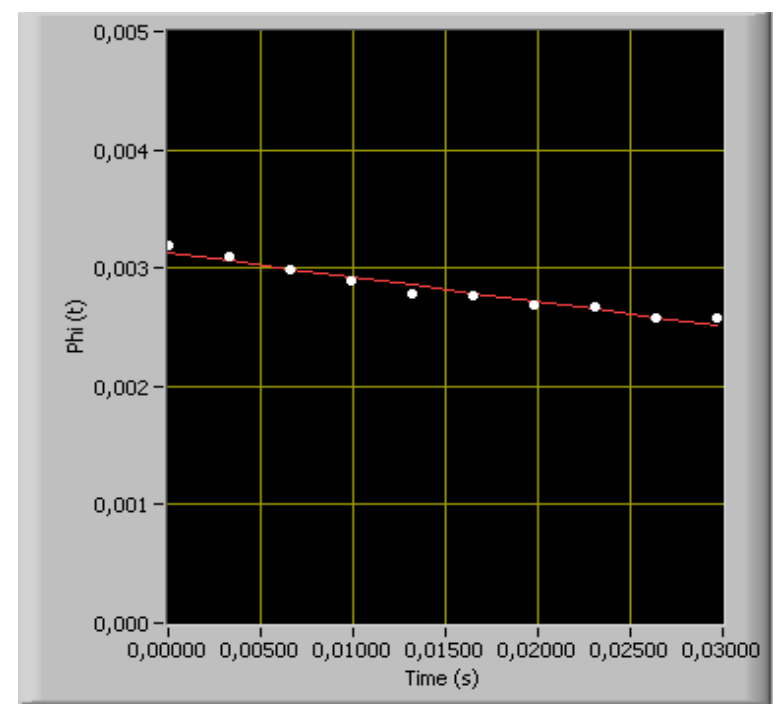

Fig. 4. $\varphi(t)$ array plot indicating by the dots and the linear fit by the red line

With the thermal diffusivity values for each frame as function of the temperature, the software make the thermal diffusivity average for intervals of $10{ }^{\circ} \mathrm{C}$ and plot this data. After all the software save all data in a file .xls and it is ready to start again.

\section{Results and discussion}

The CZ ThermaDiff software developed in this work is a prototype, because the idea is to the software acquires the image sequence and process it. But for this prototype the aim is just to prove that the method of thermal diffusivity calculation is correct and if the LabView is an appropriate tool for this work. So for this first version we used software ThermaCam Researcher (software provided by FLIR) acquire the image sequence. Therefore the control panel is shown in figure 5: 


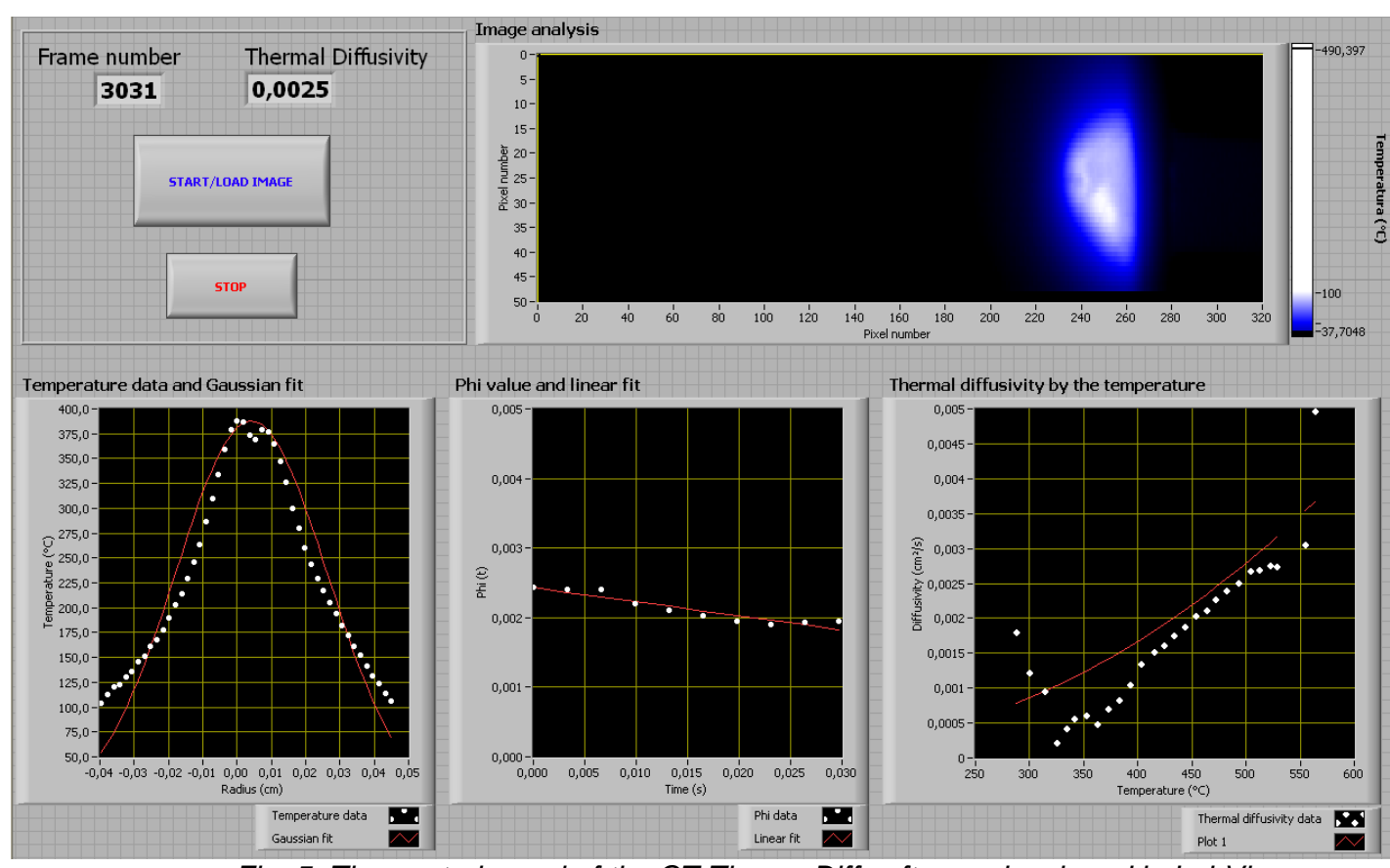

Fig. 5. The control panel of the CZ ThermaDiff software developed in LabView

The results of this work, the thermal diffusivity of enamel are shown in the figure 6 . Where it is plotted the mean values of the thermal diffusivity $\left(\mathrm{cm}^{2} / \mathrm{s}\right)$ as a function of temperature $\left({ }^{\circ} \mathrm{C}\right)$.

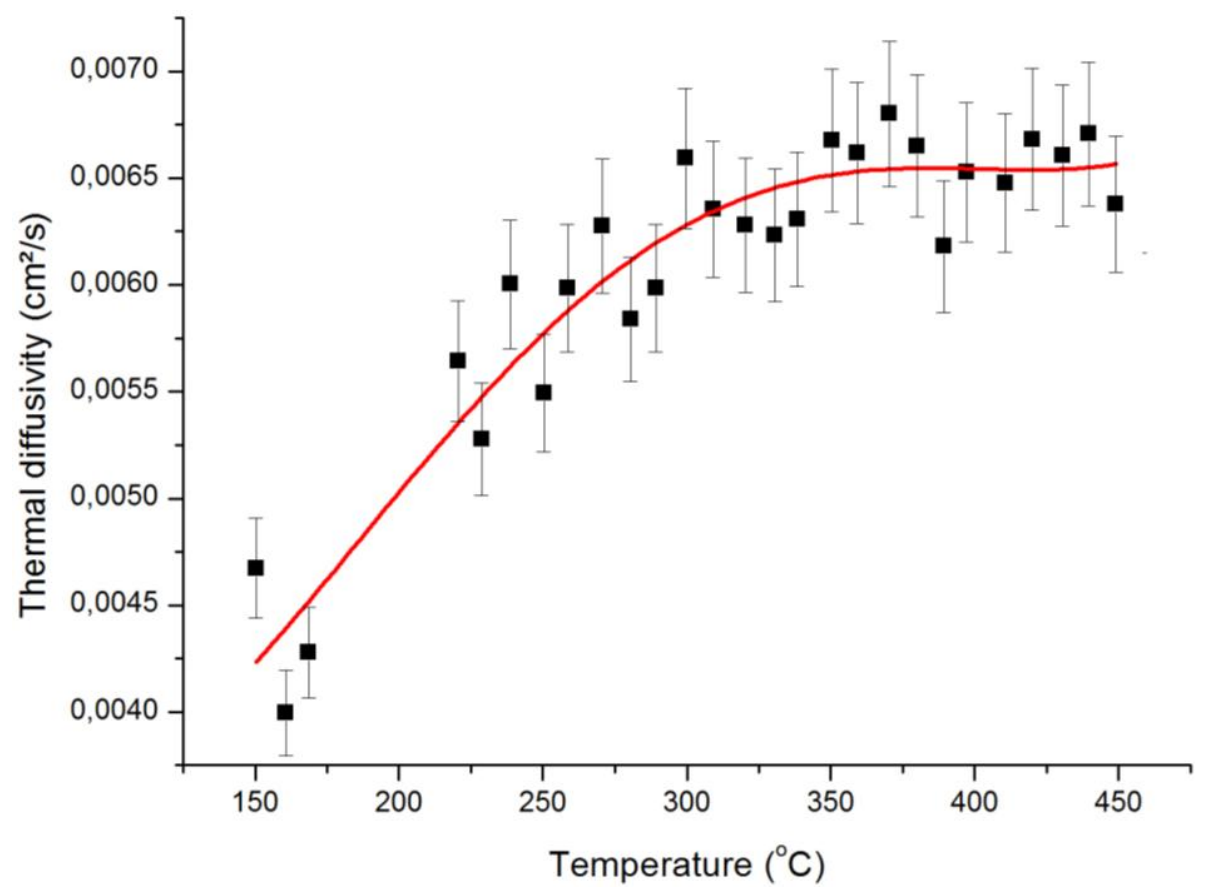

Fig. 6. This figure shows the thermal diffusivity mean as function of temperature of the enamel.

The enamel's thermal diffusivity in figure 6 starts with the values similar to those reported in the literature between 0.0041 to $0.0048 \mathrm{~cm}^{2} / \mathrm{s}[12,13,14]$, although in our results is possible to see that the thermal diffusivity to enamel, for temperatures higher than $375{ }^{\circ} \mathrm{C}$, increase reaching a value around of $0.0065 \mathrm{~cm}^{2} / \mathrm{s}$. That change happens due to in high temperatures that makes the enamel's water evaporated and the physical and chemical properties changes [10].

There are some observations that have to be made, the main problem to calculate the thermal diffusivity is the beginning and the end of the image sequence. The beginning have a problem that is the few pixels with temperature higher than the basal temperature, which makes the Gaussian fit does not work correctly. And in the end of image sequence the material deterioration and the material ablation could disturb thermal diffusivity calculation. 


\section{Conclusion}

The preliminaries results had shown that the infrared thermography method is a valid method to calculate the thermal diffusivity of enamel. The software CZ ThermaDiff created in LabView is a precise and easy tool to any user. Although that is just the first step, this because the software have some improves that will make it work better and faster than now, for example of improving is the buying of new frame-grabber from National Instruments and improve the method of image processing. And we plan that the software will be already fully integrated with the thermographic camera, determining the thermal diffusivity for different materials and dispose the experimental setup and the software CZ ThermaDiff for any laboratory.

\section{Acknowledgments}

The authors acknowledge the financial support of FAPESP CEPID (05/51689-2), National Institute of Photonic INFO/CNPq (573916/2008-0) and CAPES (PROEX).

\section{REFERENCES}

[1] Sagi-Dolev A.M., Shitzer A., Katzir A., Akselrod S., "Heating of biological tissue by laser irradiation: theoretical model". Optical Engineering Journal, vol. 31, pp. 1417-1424, 1992.

[2] Gutknecht N., Franzen R., Meister J., Vanweersch L., Maziar M., "Temperature evolution on human teeth root surface after laser assisted endodontic treatment”. Lasers in Medical Science, vol. 20, pp. 99-103, 2005.

[3] Ten Cate A.R., Dale A.C., "Oral histology : development, structure, and function”. St. Louis: Mosby, 1980.

[4] Ana P.A., Way A., Miyakawa W., Zezell D.M., "Thermal analysis of teeth irradiated with Er,Cr: YSGG at low fluences". Laser Phys. Lett, vol. 4, pp. 827-834, 2007.

[5] Pichardo-Molina J.L., Gutierrez-Juarez G., Huerta-Franco R., Vargas-Luna I., Cholico P.; Alvarado-Gil J.J., "Open photoacoustic cell technique as a tool for thermal and thermo-mechanical characterization of teeth and their restorative materials". International Journal of Thermophysics, vol. 26, pp. 243-253, 2005,

[6] Bodzenta J., Burak B., Nowak M., Pyka M. Szalajko M., Tanasiewicz M., "Measurement of the thermal diffusivity of dental filling materials using modified Angstrom's method”. Dent Mater, vol. 22, pp. 617-621, 2006.

[7] Magalhaes M.F., Ferreira R.A.N., Grossi P.A., de Andrade R.M., "Measurement of thermophysical properties of human dentin: Effect of open porosity”. Journal of Dentistry, vol. 36, pp. 588-594, 2008.

[8] Cernuschi F., Russo A., Lorenzoni L., Figari A., "In-plane thermal diffusivity evaluation by infrared thermography". Review of Scientific Instruments, vol. 72, pp. 3988-3995, 2001,.

[9] Pereira T. M., "Thermal diffusivity determination of enamel and dentin as a function of the temperature, using usin infrared thermography". University of São Paulo, Master's thesis, 2009.

[10] Antunes A., de Rossi W., Zezell D.M., "Spectroscopic alterations on enamel and dentin after nanosecond Nd: YAG laser irradiation". Spectrochimica Acta Part A Molecular and Biomolecular Spectroscopy, vol. 64, pp. 1142-1146, 2006.

[11] Johnson G. W., "LabVIEW Graphical Programming: Practical Applications in Instrumentation and Control". McGraw-Hill School Education Group, 1997.

[12] Craig R.G., Peyton F.A. "Thermal Conductivity of Tooth Structure, Dental Cements, and Amalgam". Journal of Dental Research, vol. 40, n. 3, pp. 411-418, 1961.

[13] Braden M. "Heat Conduction in Teeth + Effect of Lining Materials". Journal of Dental Research, vol. 43, n. 3, pp. 315-322, 1964.

[14] Panas A.J., Zmuda S., Terpilowski J., Preiskorn M. "Investigation Of The Thermal Diffusivity Of Human Tooth Hard Tissue". International Journal of Thermophysics, vol. 24, n. 3, pp. 837-848, 2003. 